\title{
Nanocavity enhanced diamond nitrogen-vacancy center zero phonon line emission
}

\author{
Paul E. Barclay ${ }^{1,2,3}$, Kai-Mei C. Fu ${ }^{3,4}$, Andrei Faraon ${ }^{3}$, Charles Santori ${ }^{3}$ and \\ Raymond G. Beausoleil ${ }^{3}$ \\ 1: University of Calgary, 2500 University Drive NW, Calgary, AB, T2N 1N3, Canada \\ 2: NRC-National Institute for Nanotechnology, 11421 Saskatchewan Drive NW, Edmonton, AB T6G 2M9, Canada \\ 3: Hewlett-Packard Laboratories, 1501 Page Mill Road, Palo Alto, CA 94304 USA \\ 4: University of Washington, 4014 University Way NE, Seattle, WA 98105 USA
}

\begin{abstract}
Resonantly enhanced emission of the zero phonon line of a diamond nitrogen-vacancy center in single crystal diamond is demonstrated experimentally using a hybrid whispering gallery mode nanocavity.

(c) 2011 Optical Society of America

OCIS codes: (270.0270) Quantum optics, (140.3945) Microcavities
\end{abstract}

The diamond nitrogen-vacancy (NV) center is an optically active impurity which combines many of the desirable properties of quantum dots and laser trapped atoms. Optical transitions of diamond NV centers can display low inhomogeneous broadening, and have been used to generate single photons [1], manipulate and readout single electron spins [2], and control nearby nuclear spin impurities [3, 4, 5]. Remarkably, room temperature electron spin coherence times of NVs can exceed a ms [6]. These properties make NVs a promising qubit for proposed quantum networks [7], and an attractive system for applications such as magnetometry [8] and low power optical switching [9].
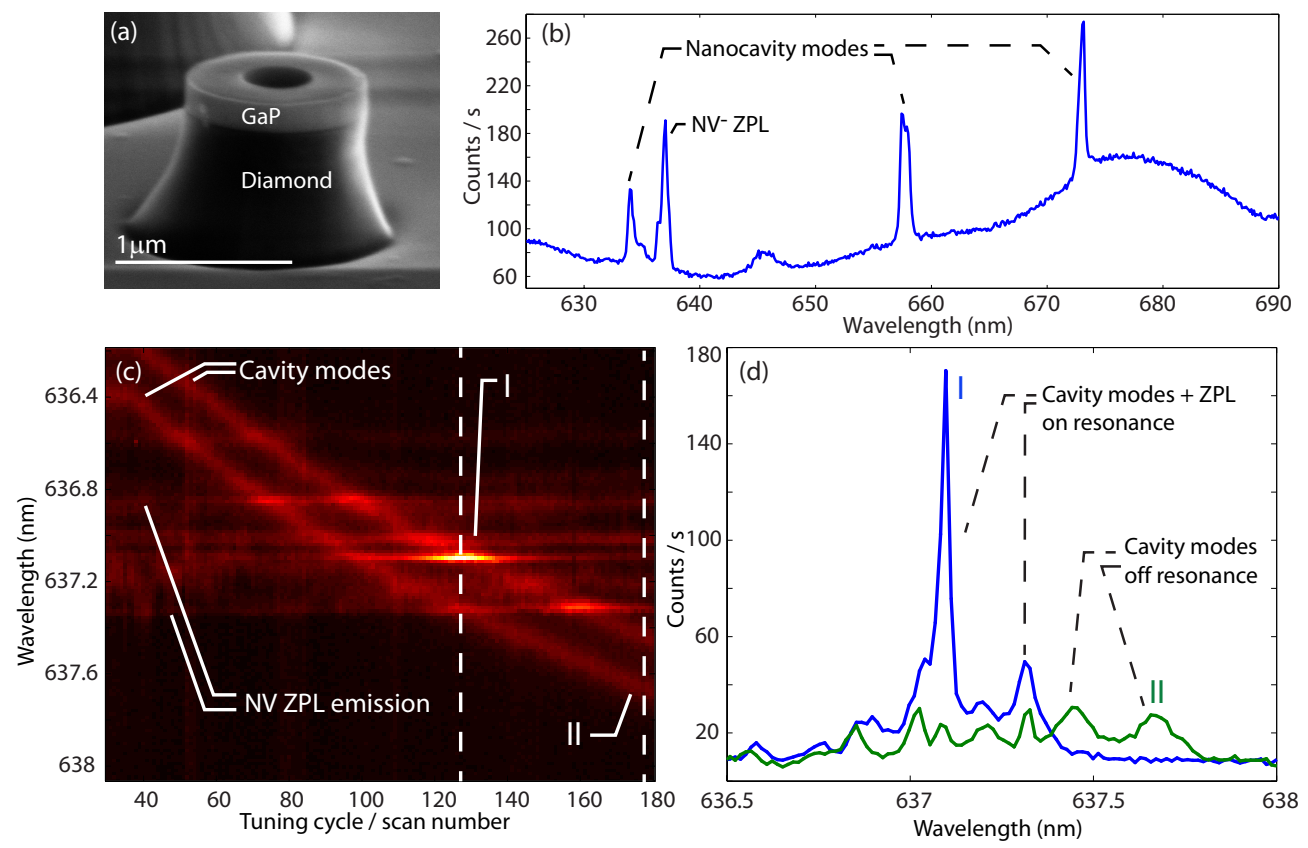

Fig. 1. (a) SEM image of a hybrid GaP-diamond whispering gallery mode nanocavity. (b) Low resolution spectra of the nanocavity studied in (c,d), prior to tuning the nanocavity modes. (c) Nanocavity PL spectra as a function of cavity tuning. (d) Nanocavity PL spectra when the nanocavity is on resonance (spectra I) and off resonance (spectra II) with the ZPL of a coupled NV. Spectra I and II are measured after the tuning cycles indicated by the dashed lines in (c). All spectra measured at $6 \mathrm{~K}$ in an He cryostat using an NA 0.45 excitation and collection objective.

Coupling NVs to nanophotonic devices promises improved efficiency for optically controlling NVs, and may enable the scalable optical coupling between multiple NVs necessary for proposed quantum information processing architectures [10]. Recent efforts to efficiently couple NVs in nanocrystalline diamond to photonic structures [11, 12, 13] have been limited by poor NV optical properties in nanocrystals compared to those found in single crystal diamond. Here we demonstrate enhanced resonant optical emission from NVs which are positioned near the surface of a single crystal diamond sample, where they are evanescently coupled to on-chip whispering gallery mode nanocavities. 
The nanocavities studied here were realized from a hybrid geometry consisting of a high refractive index gallium phosphide (GaP, 250nm thickness) whispering gallery mode nanocavity supported by a single crystal diamond substrate. A typical device is shown in Fig. 1(a). They were fabricated following the process in Ref. [14]. The device diameter, $d \sim 950 \mathrm{~nm}$, is $5 \mathrm{X}$ smaller than in previous work [14]. The diamond substrate consists of a CVD grown electronic grade single crystal diamond sample (Element Six) subjected to ion implantation $\left(\mathrm{N}^{+} 10 \mathrm{keV}, 2 \times 10^{10} \mathrm{~cm}^{-2}\right)$ and annealing $\left(950^{\circ} \mathrm{C}\right.$ in $\left.\mathrm{H}_{2} / \mathrm{Ar}\right)$ to create $\mathrm{NVs}$ close to the diamond surface. A subsequent oxygen anneal step maximized the ratio of negatively charged NVs near the surface [15]. The GaP sidewalls were extended into the diamond with an oxygen plasma etch. The nanocavity optical properties were studied by exciting the device with a $532 \mathrm{~nm}$ laser $(1-2 \mathrm{~mW})$ and measuring the resulting photoluminescence (PL) spectra. For the implantation dose used here, the NV density is such that a small number $(\sim 2-10)$ of NVs were typically excited by the laser spot. Fig. 1(b) shows a broad wavelength spectra of the nanocavity studied here. Emission from the $637 \mathrm{~nm}$ zero phonon line (ZPL) of negatively charged NVs is clearly visible. Nanocavity modes with $Q \sim 6000$ near $637 \mathrm{~nm}$ are also evident. For this device, surface roughness or elliptical deformation of the nanocavity causes the modes to form doublets corresponding to spatially orthogonal standing waves. From finite difference time domain simulations, we expect the resonances near $637 \mathrm{~nm}$ to have a mode volume of $V \sim 2.5\left(\lambda / n_{\mathrm{GaP}}\right)^{3}$ and a peak intensity in the diamond of 0.12 of the max intensity.

When this high- $Q / V$ nanocavity mode is resonant with the ZPL of an evanescently coupled NV, the ZPL spontaneous emission rate can be enhanced through the Purcell effect. In Fig. 1(c), we show the PL spectra for varying nanocavity mode wavelength. The nanocavity modes are tuned by injecting Xe gas into the cryostat. The Xe condenses on the nanocavity surface and red-shifts the nanocavity wavelength. ZPL emission between $636.8 \mathrm{~nm}-637.4 \mathrm{~nm}$ from several distinct NVs is visible. This distribution of ZPL wavelengths is possibly the result of residual strain from the implantation step. When the nanocavity modes cross the ZPL lines, in some cases the ZPL intensity increases. In particular, a large enhancement is visible when the shorter wavelength nanocavity mode is resonant with a ZPL at $637.1 \mathrm{~nm}$. The relative magnitude of this enhancement is shown in Fig. 1(d), which compares the PL spectra when the nanocavity mode is on-resonance with the ZPL of interest, with the PL spectra when it is detuned. In general, the magnitude of the enhancement depends on the NV's position and dipole orientation relative to the nanocavity field maximum. To quantitatively determine the degree of enhancement, the effect of the nanocavity mode on the spontaneous emission lifetime can be measured; this is the subject of future work, and will be discussed in our presentation. For an ideally positioned NV, we expect that a 30X ZPL emission rate enhancement can be realized with this nanocavity.

\section{References}

[1] C. Kurtsiefer, S. Mayer, P. Zarda, and H. Weinfurter, "Stable solid-state source of single photons," Phys. Rev. Lett. 85, 290 (2000).

[2] F. Jelezko, T. Gaebel, I. Popa, A. Gruber, and J. Wrachtrup, "Observation of Coherent Oscillations in a Single Electron Spin," Phys. Rev. Lett. 92, 076401 (2004).

[3] F. Jelezko, T. Gaebel, I. Popa, M. Domhan, A. Gruber, and J. Wrachtrup, "Observation of Coherent Oscillation of a Single Nuclear Spin and Realization of a Two-Qubit Conditional Quantum Gate," Phys. Rev. Lett. 93, 130501 (2004).

[4] L. Childress, M. V. Gurudev Dutt, J. M. Taylor, A. S. Zibrov, F. Jelezko, J. Wrachtrup, P. R. Hemmer, and M. D. Lukin, "Coherent Dynamics of Coupled Electron and Nuclear Spin Qubits in Diamond," Science 314, 281-285 (2006).

[5] M. V. Gurudev Dutt, L. Childress, L. Jiang, E. Togan, J. Maze, F. Jelezko, A. S. Zibrov, P. R. Hemmer, and M. D. Lukin, "Quantum Register Based on Individual Electronic and Nuclear Spin Qubits in Diamond," Science 316, 1312-1316 (2007).

[6] G. Balasubramanian, P. Neumann, D. Twitchen, M. Markham, R. Kolesov, N. Mizuochi, J. Isoya, J. Achard, J. Beck, J. Tissler, et al., "Ultralong spin coherence time in isotopically engineered diamond," Nature Materials 8, 383-387 (2009).

[7] H. Kimble, "The quantum internet," Nature 453, 1023-1030 (2008).

[8] J. R. Maze, P. L. Stanwix, J. S. Hodges, S. Hong, J. M. Taylor, P. Cappellaro, L. Jiang, M. V. G. Dutt, E. Togan, A. S. Zibrov, A. Yacoby, R. L. Walsworth, and M. D. Lukin, "Nanoscale magnetic sensing with an individual electronic spin in diamond," Nature 455, 644-647 (2008).

[9] H. Mabuchi, "Cavity-QED models of switches for attojoule-scale nanophotonic logic," Phys. Rev. A 80, 45802 (2009).

[10] S. C. Benjamin, B. W. Lovett, and J. M. Smith, "Prospects for measurement-based quantum computing with solid state spins," Laser Photonics Rev. 3, 556 (2009).

[11] P. E. Barclay, O. Painter, C. Santori, K.-M. Fu, and R. Beausoleil, "Coherent interference effects in a nano-assembled optical cavity-QED system," Opt. Express 19, 8081 (2009).

[12] D. Englund, B. Shields, K. Rivoire, F. Hatami, J. Vuckovic, H. Park, and M. D. Lukin, "Deterministic Coupling of a Single Nitrogen Vacancy Center to a Photonic Crystal Cavity," Nano Letters 10, 3922-3926 (2010).

[13] J. Wolters, A. W. Schell, G. Kewes, N. Nüsse, M. Schoengen, H. Döscher, T. Hannappel, B. Löchel, M. Barth, and O. Benson, "Enhancement of the zero phonon line emission from a single nitrogen vacancy center in a nanodiamond via coupling to a photonic crystal cavity," Applied Physics Letters 97, 141108 (2010).

[14] P. E. Barclay, K.-M. Fu, C. Santori, and R. Beausoleil, "Chip-based microcavities coupled to NV centers in single crystal diamond," Appl. Phys. Lett. 95, 191115 (2009).

[15] K. Fu, C. Santori, P. Barclay, and R. Beausoleil, "Conversion of neutral nitrogen-vacancy centers to negatively charged nitrogen-vacancy centers through selective oxidation,” Appl. Phys. Lett. 96, 121907 (2010). 